\title{
Interthalamic adhesion size in aging dogs with presumptive spontaneous brain microhemorrhages: a comparative retrospective MRI study of dogs with and without evidence of canine cognitive dysfunction
} \author{
O'Donnell $^{2}$, Simon Platt ${ }^{4}$, Kelsey Robinson ${ }^{4}$ \\ ${ }^{1}$ Clinical Sciences, Cornell University College of Veterinary Medicine, Ithaca, New York, United States \\ 2 Long Island Veterinary Specialists, Plainview, New York, United States \\ 3 Rochester Veterinary Specialists and Emergency Services, Rochester, New York, United States \\ 4 Department of Small Animal Medicine and Surgery, University of Georgia, Athens, Georgia, United States \\ Corresponding Author: Curtis W Dewey \\ Email address: cwd27@cornell.edu
}

Curtis W Dewey ${ }^{\text {Corresp., } 1,2,3 \text {, Mark Rishniw }}{ }^{1}$, Philippa J Johnson ${ }^{1}$, Emma S Davies ${ }^{1}$, Joseph J Sackman ${ }^{2}$, Marissa

Objective: Spontaneous brain microhemorrhages in elderly people are present to some degree in Alzheimer's disease patients but have been linked to brain atrophy in the absence of obvious cognitive decline. Brain microhemorrhages have recently been described in older dogs, but it is unclear whether these are associated with brain atrophy. Diminution of interthalamic adhesion size-as measured on MRI or CT-has been shown to be a reliable indicator of brain atrophy in dogs with canine cognitive dysfunction (CCD) in comparison with successfully aging dogs. We hypothesized that aging dogs with brain microhemorrhages presenting for neurologic dysfunction but without obvious features of cognitive decline would have small interthalamic adhesion measurements, like dogs with CCD, compared with control dogs. The objective of this study was to compare interthalamic adhesion size between three groups of aging (> 9 yrs) dogs: 1) neurologically impaired dogs with presumptive spontaneous brain microhemorrhages and no clinical evidence of cognitive dysfunction 2) dogs with canine cognitive dysfunction 3) dogs without clinical evidence of encephalopathy on neurologic examination (control dogs). MR images from 52 aging dogs were reviewed and measurements were obtained of interthalamic adhesion height (thickness) and mid-sagittal interthalamic adhesion area for all dogs, in addition to total brain volume. Interthalamic adhesion measurements, either absolute or normalized to total brain volume were compared between groups. Signalment (age, breed, sex), body weight, presence and number of SBMs, as well as other abnormal MRI findings were recorded for all dogs. Results: All interthalamic adhesion measurement parameters were significantly $(p<0.05)$ different between control dogs and affected dogs. 
Both dogs with cognitive dysfunction (12/15; $80 \%$ ) and dogs with isolated brain microhemorrhages had more microhemorrhages than control dogs $(3 / 25 ; 12 \%)$. Affected dogs without cognitive dysfunction had significantly more microhemorrhages than dogs with cognitive dysfunction. In addition to signs of cognitive impairment for the CCD group, main clinical complaints for SBM and CCD dogs were referable to central vestibular dysfunction, recent-onset seizure activity, or both. Geriatric dogs with spontaneous brain microhemorrhages without cognitive dysfunction have similar MRI abnormalities as dogs with cognitive dysfunction but may represent a distinct disease category. 
1 ORIGINAL RESEARCH ARTICLE: Peer J

2 TITLE: INTERTHALAMIC ADHESION SIZE IN AGING DOGS WITH PRESUMPTIVE

3 SPONTANEOUS BRAIN MICROHEMORRHAGES: A COMPARATIVE

4 RETROSPECTIVE MRI STUDY OF DOGS WITH AND WITHOUT EVIDENCE OF

5 CANINE COGNITIVE DYSFUNCTION

6 AUTHORS NAMES AND DEGREES:

7 Curtis Wells Dewey ${ }^{1-3}$, DVM, MS, CTCVMP, DACVIM (Neurology), DACVS; Mark Rishniw ${ }^{1}$,

8 BVSc, MS, PhD, DACVIM (Internal Medicine, Cardiology); Philippa J. Johnson ${ }^{1}$, BVSc,

9 CertVDI, DECVDI, MSc, MRCVS; Emma S. Davies¹, BVSc, MSc, DECVN; Joseph Sackman ${ }^{3}$,

10 AA, BS; Marissa O’Donnell'3; Simon Platt ${ }^{4}$, BVM\&S, FRCVS, DACVIM (Neurology),

11 DECVN; Kelsey Robinson ${ }^{4}$, DVM

AUTHOR AFFILIATIONS: Clinical Sciences ${ }^{1}$, College of Veterinary Medicine, Cornell University, Ithaca, New York, United States; Rochester Veterinary Specialists and Emergency Services $^{2}$, Rochester, New York, United States; Long Island Veterinary Specialists ${ }^{3}$, Plainview, New York, United States; Department of Small Animal Medicine and Surgery ${ }^{4}$, University of Georgia, Athens, Georgia, United States.

\section{AUTHOR CONTACT INFORMATION:}

CW Dewey: C4 169 Clinical Programs Center, Cornell University College of Veterinary Medicine, Ithaca, NY 14853; cwd27@,cornell.edu

M Rishniw: C2 015 Clinical Programs Center, Cornell University College of Veterinary Medicine, Ithaca, NY 14853; mr89@,cornell.edu 
22 PJ Johnson: Department of Clinical Sciences, Cornell University College of Veterinary

23 Medicine, 930 Campus Road, Box 25, Ithaca, NY 14853; pjj43@cornell.edu

24 ES Davies: C4 107 Clinical Programs Center, Cornell University College of Veterinary

25 Medicine, Ithaca, NY 14853; ed445@,cornell.edu

26 J Sackman: Long Island Veterinary Specialists, 163 South Service Road, Plainview, NY 11803;

27 jsackman@livs.org.

28 M O’Donnell: Long Island Veterinary Specialists, 163 South Service Road, Plainview, NY

29 11803; modonnell@livs.org.

30 SR Platt: Department of Small Animal Medicine \& Surgery, College of Veterinary Medicine,

31 University of Georgia, 2200 College Station Rd, Athens, GA 30602; srplatt@uga.edu

32 K Robinson: Department of Small Animal Medicine \& Surgery, College of Veterinary Medicine,

33 University of Georgia, 2200 College Station Rd, Athens, GA 30602; k.robinson@uga.edu

34 CORRESPONDING AUTHOR: Curtis W Dewey: C4 169 Clinical Programs Center, Cornell

35 University College of Veterinary Medicine, Ithaca, NY 14853; cwd27@cornell.edu 
38

39

40

41

42 57 dogs.

\section{ABSTRACT}

Objective: Spontaneous brain microhemorrhages in elderly people are present to some degree in Alzheimer's disease patients but have been linked to brain atrophy in the absence of obvious cognitive decline. Brain microhemorrhages have recently been described in older dogs, but it is unclear whether these are associated with brain atrophy. Diminution of interthalamic adhesion size-as measured on MRI or CT-has been shown to be a reliable indicator of brain atrophy in dogs with canine cognitive dysfunction (CCD) in comparison with successfully aging dogs. We hypothesized that aging dogs with brain microhemorrhages presenting for neurologic dysfunction but without obvious features of cognitive decline would have small interthalamic adhesion measurements, like dogs with CCD, compared with control dogs. The objective of this study was to compare interthalamic adhesion size between three groups of aging ( $\geq 9$ yrs) dogs: 1) neurologically impaired dogs with presumptive spontaneous brain microhemorrhages and no clinical evidence of cognitive dysfunction 2) dogs with canine cognitive dysfunction 3) dogs without clinical evidence of encephalopathy on neurologic examination (control dogs). MR images from 52 aging dogs were reviewed and measurements were obtained of interthalamic adhesion height (thickness) and mid-sagittal interthalamic adhesion area for all dogs, in addition to total brain volume. Interthalamic adhesion measurements, either absolute or normalized to total brain volume were compared between groups. Signalment (age, breed, sex), body weight, presence and number of SBMs, as well as other abnormal MRI findings were recorded for all

Results: All interthalamic adhesion measurement parameters were significantly $(\mathrm{p}<0.05)$ different between control dogs and affected dogs. Both dogs with cognitive dysfunction (12/15; $80 \%$ ) and dogs with isolated brain microhemorrhages had more microhemorrhages than control 
61 dogs $(3 / 25 ; 12 \%)$. Affected dogs without cognitive dysfunction had significantly more

62 microhemorrhages than dogs with cognitive dysfunction. In addition to signs of cognitive

63 impairment for the CCD group, main clinical complaints for SBM and CCD dogs were referable

64 to central vestibular dysfunction, recent-onset seizure activity, or both. Geriatric dogs with

65 spontaneous brain microhemorrhages without cognitive dysfunction have similar MRI

66 abnormalities as dogs with cognitive dysfunction but may represent a distinct disease category.

67 Keywords: Canine, dog, brain, microhemorrhage, cognitive, dementia, MRI, beta-amyloid,

68 Alzheimer's, cerebral amyloid angiopathy 


\section{Introduction}

70 Geriatric humans can suffer brain microhemorrhages, often referred to as cerebral

71 microhemorrhages or cerebral microbleeds. Brain microhemorrhages can occur secondary to

72 several disorders, including neoplasia, coagulopathies and hypertensive states (e.g., renalassociated hypertension). ${ }^{1,2}$ These microhemorrhages can also occur as a result of cerebral amyloid angiopathy, which is a specific cerebral vasculopathy of geriatric humans caused by the accumulation of $\beta$-amyloid protein in vessel walls of brain arterioles and capillaries. Microbleeds are often detected by MRI in geriatric human patients with a variety of underlying brain disorders, including Alzheimer's disease and cerebral amyloid angiopathy, and, in 5\% to 15\% of geriatric humans, as incidental findings. ${ }^{1-5}$ Microbleeds in humans appear on MRI as circular, ovoid or "dot-like" parenchymal lesions, best identified with T2* gradient echo or susceptibilityweighted MR sequences. Although there is some variability in the literature concerning what constitutes a microhemorrhage, most reports include lesions that are less than $5.0 \mathrm{~mm}$ in diameter. ${ }^{1-5}$ Pathologists have identified both cerebral amyloid angiopathy and cerebral microbleeds in geriatric dogs ${ }^{9-15}$, and clinicians have described putative microhemorrhages in geriatric dogs undergoing MRI. ${ }^{16-18}$

Anecdotally, we have observed geriatric dogs with and without evidence of cognitive dysfunction occasionally present with recent onset seizure activity and/or central vestibular dysfunction. The vestibular dysfunction in these dogs is generally much milder than that displayed in dogs with "geriatric vestibular syndrome" and tends to improve more rapidly than the peripheral disorder. These dogs typically exhibit varying numbers of punctuate lesions on MRI, consistent with microbleeds or microhemorrhages described in humans. As with human patients with spontaneous brain microhemorrhages, veterinary neurologists often fail to identify 
92 any metabolic cause for microhemorrhages in geriatric dogs. In one study of putative

93 microhemorrhages in dogs, an association was found between systemic hypertension and the

94 presence of brain microhemorrhages ${ }^{18}$; however, systemic hypertension can be a result of brain

95 pathology as well as causing brain pathology, as the control center for systemic blood pressure is

96 located in the medulla and is responsive to changes in cerebral oxygen levels. ${ }^{19}$

97 Dogs with cognitive dysfunction have smaller interthalamic adhesion heights than similarly aged

98 control dogs without cognitive dysfunction. ${ }^{20,21}$ This change is not considered specific for CCD

99 but is indicative of a generalized neurodegenerative process. ${ }^{20}$

100 We hypothesized that aging dogs with cognitive dysfunction and dogs presenting with acute

101 neurological signs that cannot be attributed to intracranial neoplasia, hypertensive or

102 coagulopathic intracranial hemorrhage, but without evidence of cognitive dysfunction, often

103 have lesions consistent with spontaneous brain microhemorrhages detected on MRI, compared to 104 similarly aged dogs without encephalopathy or cognitive dysfunction. We further hypothesized 105 that dogs with cognitive dysfunction, and dogs without cognitive dysfunction but with evidence 106 of spontaneous brain microhemorrhages, would have smaller interthalamic adhesion height and 107 area than similarly aged control dogs. 
110 We identified the dogs for our study using three strategies. For the control dogs, we searched

111 medical records for older ( $\geq 9 \mathrm{yrs}$ ) dogs who had brain MRIs performed but had no clinical signs

112 of encephalopathy on neurologic examination (e.g., nasal disease, peripheral vestibular disease).

113 Because few geriatric dogs without clinical evidence of brain disease undergo brain MRI, we 114 acquired additional control geriatric brain MR images from two sources: 10 mixed-breed retired

115 sled dogs with normal neurologic examinations that had been imaged as part of another study 116 and 6 neurologically normal small-breed dogs whose owners volunteered for a no-cost brain

117 MRI prior to scheduled dentistry procedures. In addition to being 9 yrs or older, control dogs

118 were included if they had no historical or clinical evidence suggesting structural brain disease, 119 and no conditions that might predispose to spontaneous brain hemorrhages (e.g. hypertension, 120 coagulopathy).

121 For dogs with cognitive dysfunction, we searched medical records for dogs with a clinical

122 diagnosis of cognitive dysfunction that had undergone MRI. We based our diagnosis of cognitive 123 dysfunction on previously established historical and clinical findings in association with

124 characteristic MRI abnormalities. ${ }^{22-25}$ We only included cases of canine cognitive dysfunction for 125 which this diagnosis was clearly stated in the medical record and supported by the MRI report.

126 For dogs with spontaneous brain hemorrhages, we searched the medical records for dogs that 127 had undergone MRI for investigation of encephalopathic signs and had an imaging diagnosis of 128 brain microhemorrhages assigned. We defined spontaneous brain microhemorrhages using the 129 same criteria as had been previously proposed in humans and dogs; namely, as punctate areas of 130 signal void in the brain parenchyma of dogs (Figure 1) with no underlying metabolic reason 131 (e.g., coagulopathy, endocrine disorder, renal hypertension, vascular neoplasia, etc.) or 
132 associated intracranial reason (e.g., tumor, inflammatory brain disease, etc.) for intracranial

133 bleeding. ${ }^{5-7,18}$

134 We excluded any dogs that had macrohemorrhages, or evidence of disorders that could cause

135 brain hemorrhages, such as systemic hypertension, coagulopathies, intracranial neoplasia etc.

136 We searched databases from five institutions (Cornell University Hospital for Animals, Long

137 Island Veterinary Specialists, Rochester Veterinary Specialists and Emergency Services,

138 University of Georgia Small Animal Hospital and Oradell Animal Hospital) from 2010 to 2019.

All MRIs were performed under general anesthesia with one of six magnets: 1) $1.5 \mathrm{~T}$

Siemens Avanto (Munich, Germany) 2) 1.5 T Toshiba Vantage Elan (Lake Forest CA, USA) 3) Imaging sequences acquired included the following: sagittal T2-weighted; transverse T2-and T1weighted; transverse and dorsal plane T1-weighted post-gadolinium injection; transverse T2fluid attenuated inversion recovery (FLAIR); and transverse T2* gradient-recalled echo (GRE).

145 For the 1.5-T MRI units, measurement parameters were as follows: slice thickness, $3.5 \mathrm{~mm}$; slice gap, $3.5 \mathrm{~mm}$; FOV, $185 \mathrm{~mm}$; matrix size of images, $480 \times 480$. For the 3.0-T MRI units, measurement parameters were as follows: slice thickness, $2.0 \mathrm{~mm}$; slice gap, 1.0-3.0 mm 148 (depending on dog size); FOV, 1101 mm; matrix size of images, 400 x 400.

149 For each dog, two investigators (JS \& MO) measured the interthalamic adhesion height on transverse T2-weighted images, as previously described (Figure 2). ${ }^{20}$ Additionally, these

151 investigators measured the cross-sectional area of the interthalamic adhesion on mid-sagittal T2-

152 weighted images (Figure 3), and total brain volume for each dog, using Mimics ${ }^{\circledR}$ software.

153 Volumetric measurements for total brain volume were performed as previously described. ${ }^{26}$ 
154 Both investigators used previously published anatomic landmarks for all measurements ${ }^{27}$ and

155 both were blinded to the clinical status of the dogs in the study. One investigator counted all

156 microhemorrhages evident on MR images for each dog (CD), based on transverse T2* gradient

157 echo sequences.

\section{Statistical analysis}

159 We used Kruskal Wallis tests, with subsequent multiple comparisons by the Conover method for

160 all comparisons described below. We did not correct for any experiment-wise error rate.

161 We first compared ages and body weights of the three groups of dogs to demonstrate similarity

162 of cohorts.

163 We compared the heights and areas of the interthalamic adhesions between the three groups of

164 dogs both as absolute values, and after indexing to total brain volume (under the assumption that

165 the height of the interthalamic adhesion would be proportional to the size of the brain).

166 Finally, we compared the number of microhemorrhages between the three groups of dogs.

167 To examine the reproducibility of the measurements of interthalamic adhesion height and area,

168 one investigator re-measured these variables in 41 dogs several months after the initial

169 measurements. We compared the pairs of measurements using a Limits of Agreement approach.

170 To examine the reproducibility of microhemorrhage identification, one investigator re-counted

171 the microhemorrhages in 10 dogs with either cognitive dysfunction or spontaneous brain

172 microhemorrhages. We examined the absolute difference between the pairs of results, but

173 performed no statistical analyses. 


\section{Results}

176 Our study consisted of 52 aging dogs: 25 control dogs, 12 dogs with spontaneous brain

177 hemorrhages and neurologic impairment without evidence of cognitive dysfunction, and 15 dogs

178 with evidence of cognitive dysfunction. Control dogs were younger than affected dogs (median

179 age 11 yrs vs. 13 yrs; $P<0.0001)$. Bodyweights of dogs in each group did not differ $(P=0.9)$.

180 Breeds represented in the control dog group (CD) included mixed breed (12), Chihuahua (4),

181 Dachshund (2) and one each of Yorkshire terrier, Beagle, Boston terrier, Golden Retriever,

182 Coonhound, West Highland White terrier and Maltese. There were 9 female spayed, 3 male

183 castrated and 2 each of female and male intact dogs in the control group. Breeds represented in

184 the spontaneous brain microhemorrhage group without cognitive impairment (SBM) included

185 one each of Shih tzu (), Labrador retriever, mixed breed Maltese, Greyhound, Shetland

186 Sheepdog, Boston terrier, Chihuahua, Golden retriever, Pug, Yorkshire terrier and Bichon Frise.

187 There were 6 female spayed dogs, 5 male castrated dogs and one male intact dog. Breeds

188 represented in the cognitive dysfunction (CCD) group included Shih tzu (2), Springer spaniel (2),

189 mixed breed (2) and one each of Labrador retriever, Chihuahua, Miniature Poodle, Shetland

190 Sheepdog, Cockapoo, Samoyed, Miniature Schnauzer, Tibetan terrier, and Wheaten terrier.

191 There were 8 female spayed, 5 male castrated and 2 male intact dogs.

192 Dogs with spontaneous brain microhemorrhages presented with central vestibular dysfunction

193 (9), recent-onset seizures (2) and a combination of the two (1). Dogs with canine cognitive

194 dysfunction presented with cognitive dysfunction alone (5), recent-onset seizures (6) central

195 vestibular dysfunction (3) and non-ambulatory paraparesis (1).

196 Control dogs had taller sagittal interthalamic adhesions and larger interthalamic adhesion areas

197 than dogs with microhemorrhages without cognitive impairment and dogs with cognitive 
198 dysfunction ( $P=0.0001$ for both comparisons). Dogs with cognitive dysfunction had taller

199 sagittal interthalamic adhesions and larger interthalamic adhesion areas than dogs with

200 microhemorrhages without cognitive impairment. When indexed to total brain volume, control

201 dogs had taller sagittal interthalamic adhesions than dogs with cognitive dysfunction, but not

202 dogs with microhemorrhages without cognitive dysfunction $(P=0.03)$. However, when indexed

203 to total brain volume, control dogs had larger interthalamic adhesion areas than both groups of

204 abnormal dogs $(P=0.0001)$ (Figure 4). Dogs with spontaneous brain microhemorrhages without

205 cognitive dysfunction and those with cognitive dysfunction did not differ from each other

$206(P>0.05)$.

207

208

209

210

211

212

213

214

215

216

217 aged control dogs (Figure 6).

218

219

220

Three control dogs $(3 / 25 ; 12 \%)$ exhibited evidence of microhemorrhages, with 2 hemorrhages in one dog and a single hemorrhagic lesion in each of the other two. Twelve of 15 dogs with cognitive dysfunction ( $80 \%$ ) had evidence of brain microhemorrhages. Control dogs had fewer microhemorrhages than either group of abnormal dogs. Dogs with cognitive dysfunction had fewer microhemorrhages than dogs without cognitive dysfunction $(P<0.0001)$ (Figure 5).

When plotted against age, dogs with spontaneous brain hemorrhages and dogs with cognitive dysfunction did not display evidence of decreasing sagittal interthalamic adhesion height or interthalamic adhesion area (Figure 6). Control dogs displayed weak evidence of decreasing interthalamic adhesion height and area with age. Dogs with cognitive dysfunction tended to have smaller interthalamic adhesion height, but not interthalamic adhesion area, than similarly Duplicate measurements of interthalamic adhesion height showed no bias with $95 \%$ Limits of Agreement being $\pm 1 \mathrm{~mm}$. Similarly, duplicate measurements of interthalamic adhesion area showed no bias with 95\% Limits of Agreement being $\pm 3 \mathrm{~mm}^{2}$. 
221 Duplicate counts of microhemorrhages in 10 dogs differed by a median of 1 microhemorrhage,

222 ranging from 0 to 5 (the largest difference was in a dog with 75 microhemorrhages).

223

224

225

226

227

228

229

230

231

232

233

234

235

236

237

238

239 


\section{Discussion}

241 Our study demonstrates that neurologically impaired aging dogs with spontaneous brain

242 microhemorrhages, but without evidence of cognitive dysfunction, have MRI features similar to,

243 but less severe than dogs with cognitive dysfunction. Both groups of dogs with spontaneous

244 brain microhemorrhages had smaller normalized interthalamic adhesion areas than similarly

245 sized and aged control dogs. Additionally, dogs with cognitive dysfunction had smaller sagittal

246 interthalamic adhesion heights than control dogs. These decreases appeared to be independent of

247 age. The interthalamic adhesion measurements did not differ between the two neurologically

248 affected groups of dogs. Somewhat surprisingly, dogs without cognitive dysfunction had more

249 microhemorrhages than dogs with cognitive dysfunction. The apparent absence of cognitive

250 dysfunction in the group of dogs with nearly twice the number of microhemorrhages as in the

251 dogs with cognitive dysfunction suggests that factors other than microhemorrhages might be

252 responsible for cognitive dysfunction.

253 Our results support the observations of previous investigators, who also found smaller

254 interthalamic adhesions in dogs with cognitive dysfunction than in control dogs. ${ }^{20,21}$ However,

255 decreases in interthalamic adhesion size are not specific for cognitive dysfunction, and have been

256 reported sporadically in other disorders that can cause brain atrophy. ${ }^{20}$ We failed to see a clear

257 relationship with either interthalamic sagittal adhesion height or area and age in either of the

258 affected dog groups, suggesting that these changes are not age-related.

259 Because we could not corroborate our findings with histopathology, we can only speculate that

260 the lesions we observed on MRI are indeed microhemorrhages. However, the shape, size and

261 imaging characteristics we identified are consistent with previous reports of microhemorrhages

262 in both humans and dogs. ${ }^{1-5,16-18}$ 
263 What could be causing microhemorrhages in dogs? Currently, we can only speculate about the

264 cause. Pathologists have described histopathologic features of cerebral amyloid angiopathy in

265 dogs, and have noted the presence of cerebral hemorrhages in these cases. ${ }^{10-13}$ Therefore, we can

266 reasonably hypothesize that the microhemorrhages in our cohorts might be the result of cerebral

267 amyloid angiopathy. Similar microhemorrhages in people are characteristic for cerebral amyloid

268 angiopathy, the diagnosis of which generally relies upon MRI findings and supporting clinical

269 features. ${ }^{5-8}$ Indeed, according to the Boston criteria for diagnosing human cerebral amyloid

270 angiopathy, supportive clinical data and MRI evidence of microhemorrhages, combined with the

271 absence of any other identifiable cause for hemorrhage, supports a diagnosis of "probable

272 cerebral amyloid angiopathy" in the dogs in our study. ${ }^{6,7,28-30}$

273 Putative brain microhemorrhages in dogs were described in a previous MRI-based study. ${ }^{18}$

274 These investigators compared dogs with microhemorrhages to all other dogs undergoing similar

275 brain MRIs, regardless of the indications for pursuing imaging. They found that dogs with

276 cerebral microhemorrhages were older and smaller, and presented more frequently for vestibular

277 dysfunction than the control dogs. ${ }^{18}$ Similar to this study, our populations of dogs with

278 microhemorrhages were primarily small-breed dogs; because we selected our controls to be as

279 similar in age as possible to the dogs with cognitive dysfunction or microhemorrhages, we

280 cannot comment on the effect of age, although all of our dogs were older ( $\geq 9$ yo). It is also

281 possible that smaller breeds would be expected to predominate because they live long enough to

282 develop degenerative brain disease, compared with larger dog breeds

283 Both cerebral amyloid angiopathy and cognitive dysfunction are well-established pathologies of

284 elderly dogs. ${ }^{9-15,20-25}$ Our study suggests that the dogs with spontaneous brain microhemorrhages

285 represent a distinct neurodegenerative brain disorder with some similarities to cognitive 
286 dysfunction, but other features more reminiscent of cerebral amyloid angiopathy. Our suspicion

287 that these dogs have cerebral amyloid angiopathy will require future histopathologic examination

288 of brain tissue from patients with similar MRI abnormalities. Furthermore, our observations

289 suggest that microhemorrhages are likely not the sole cause of cognitive dysfunction in dogs.

290 Three dogs with cognitive dysfunction had no microhemorrhages and dogs without cognitive

291 dysfunction had more microhemorrhages than dogs with cognitive dysfunction. We would have

292 expected the opposite if microhemorrhages were causally associated.

293 In addition to the lack of histopathologic correlation with the imaging findings in this study,

294 there are several other limitations to our investigation, most of which are a consequence of its

295 retrospective nature. We made the diagnosis of cognitive dysfunction in all dogs via historical

296 and clinical features consistent with cognitive impairment, combined with supportive MRI

297 findings. While this manner of diagnosing cognitive dysfunction is common practice and

298 adequate for a clinical diagnosis, it is likely to under-diagnose dogs with mild to moderate

299 cognitive impairment. There are a number of accurate behavioral test protocols for dogs that

300 provide more objective data regarding cognitive health. ${ }^{22,24,31,32}$ Although the dogs with isolated

301 microhemorrhages did not appear to have cognitive dysfunction, it is possible that they exhibited

302 some level of cognitive dysfunction that was not appreciable without specific behavioral testing.

303 Because microhemorrhages are known to occur in elderly people as an incidental finding, we

304 attempted to accumulate a large cohort of aging control dogs in order to assess to what level

305 microhemorrhages may be an incidental aging finding in dogs. In addition, when searching MRI

306 databases for microhemorrhages, we intended to place any such incidental cases in the control

307 group. No such cases were identified. In addition, none of the aging control dogs derived from

308 hospital MRI records exhibited evidence of microhemorrhages. It is possible that the some of the 
309 aging control dogs that were chosen from hospital MRI records could have biased the control 310 group and underestimated the extent of incidental microhemorrhages. However, 16 control dogs

311 with no evidence of brain dysfunction were recruited for this investigation, and only 3 (19\%)

312 exhibited evidence of microhemorrhages, all 3 of whom had 1 (2 dogs) or 2 ( 1 dog) lesions. Ten

313 of the geriatric control dogs were kennel-housed and therefore not part of a home environment,

314 unlike the remainder of the control dogs and all dogs with microhemorrhages. As such, cognitive 315 dysfunction in some of these kennel-housed control dogs could have gone unrecognized. Two of

316 these dogs did have small interthalamic adhesions, despite being deemed as cognitively normal.

317 Ideally, all the control dogs would have been from home environments, in which subtle

318 behavioral changes could have been observed by owners. Although measuring interthalamic

319 adhesion thickness from a transaxial MR image slice is an accurate and easily applicable clinical

320 tool for the diagnosis of canine cognitive dysfunction ${ }^{20,21}$, some inherent error in this method

321 exists. The image slice that appears to have the largest interthalamic adhesion measurement is

322 chosen from the slices available, which introduces a level of variability in the resultant data.

323 Since the mid-sagittal interthalamic adhesion area is more consistent, this is likely a more

324 accurate mode of measuring the interthalamic adhesion. ${ }^{20}$ We found no differences between the

325 two groups of neurologically affected dogs, but the sample sizes were small and might have

326 failed to detect a difference.

327 Our main goal with this investigation was to compare interthalamic adhesion measurements

328 between two abnormal aging groups of dogs: neurologically affected dogs with presumptive

329 spontaneous brain microhemorrhages and no obvious cognitive impairment and dogs with

330 evidence of canine cognitive dysfunction (with or without spontaneous brain

331 microhemorrhages). We chose our aging control dogs based upon a lower age limit of 9 years of 
332 age. Although this lower limit is based on several reports dealing with canine cognitive

333 dysfunction $22,25,32$, the definition of what minimum age defines "aging", "older" or "geriatric" in

334 dogs is somewhat arbitrary. There are several potential interpretations of this finding. One

335 possibility is that both brain disorders investigated in this report are more likely to occur with

336 increasing age. This phenomenon has been documented with canine cognitive dysfunction. ${ }^{22-}$

$33725,33,34$ In one study of putative microhemorrhages in $\operatorname{dogs}^{18}$, affected dogs were significantly

338 older than unaffected dogs, but an increasing tendency for brain microhemorrhages to occur with

339 aging has not yet been established in this species. Another possibility is that a lower age limit of

3409 years is too low for a definition of "geriatric" in dogs. If this is the case, interthalamic adhesion

341 measurements may be less discriminative for degenerative brain pathology if the lower age limit

342 for geriatric control dogs is increased. In one study evaluating interthalamic adhesion thickness

343 as an indicator of cognitive dysfunction in dogs, 9 years of age was chosen as a lower limit for

344 defining geriatric status. These investigators found a significant difference in interthalamic

345 adhesion thickness between aging non-cognitively impaired and cognitively impaired older

346 dogs. $^{21}$

347 Future investigation into geriatric dogs with spontaneous brain microhemorrhages will hopefully

348 include correlating MR imaging findings with histopathology, as well as more objective

349 assessment of cognitive status. In addition, comparing imaging features of larger cohorts of dogs

350 with and without cognitive dysfunction might help discern whether these entities are distinct

351 with respect to interthalamic adhesion measurements.

352 In conclusion, we have associated small interthalamic adhesion size with spontaneous brain

353 microhemorrhages in neurologically impaired dogs that lack evidence of cognitive dysfunction

354 as well as in dogs with cognitive dysfunction. Dogs with microhemorrhages had smaller 
355 interthalamic adhesions than similarly aged, weight-matched control dogs. Dogs without

356 cognitive dysfunction had more hemorrhages than dogs with cognitive dysfunction, suggesting

357 that the cognitive dysfunction is not associated with hemorrhage number. Our data suggest but

358 do not confirm the idea that spontaneous brain microhemorrhages in dogs might be a

359 manifestation of amyloid angiopathy.

360 
361

362

363

364

365

366

367

368

369

370

371

372

373

374

375

376

377

378

379

380

381

382

383

\section{References}

1. Jouvent E, Puy L, Chabriat H. Cerebral microhemorrhages: significance, associations, diagnosis, and treatment. Curr Treat Options Neurol. 2016; DOI: 10.1007/s11940-0160418-1.

2. Ungvari Z, Tarantini S, Kirkpatrick AC, Csiszar A, Prodan CI. Cerebral microhemorrhages: mechanisms, consequences, and prevention. Am J Physiol Heart Circ Physiol. 2017; DOI: 10.1152/ajpheart.00780.2016.

3. Murao K, Rossi C, Cordonnier C. Intracerebral haemorrhage and cognitive decline. Revue Neurologique. 2013; 169: 772-778.

4. Bos D, Wolters FJ, Darweesh SKL, Vernooij MW, de Wolf F, Ikram MA, Hofman A. Cerebral small vessel disease and the risk of dementia: a systematic review and metaanalysis of population-based evidence. Alzheimer's \& Dementia. 2018; https://doi.org/10.1016/j.jalz.2018.04.007.

5. Sharma R, Dearaugo S, Infeld B, O’Sullivan R, Gerraty RP. Cerebral amyloid angiopathy: review of clinic-radiological features and mimics. Journal of Medical Imaging and Radiation Oncology. 2018; 62: 451-463.

6. Yamada M. Cerebral amyloid angiopathy: emerging concepts. Journal of Stroke. 2015; 17: 17-30.

7. Boulouis G, Charidimou A, Greenberg SM. Sporadic cerebral amyloid angiopathy: pathophysiology, neuroimaging features, and clinical implications. Semin Neurol. 2016; 36: $233-243$.

8. Smith EE. Cerebral amyloid angiopathy as a cause of neurodegeneration. J Neurochem. 2018; 144: 651-658. 
384

385

386

387

388

389

390

391

392

393

394

395

396

397

398

399

400

401

402

403

404

405

9. Uchida K, Nakayama H, Goto N. Pathological studies of cerebral amyloid angiopathy, senile plaques and amyloid deposition in visceral organs in aged dogs. J Vet Med Sci. 1991; 53: 1037-1042.

10. Shimada A, Kuwamura M, Awakura T, Umemura T, Takada K, Ohama E, Itakura C. Topographic relationship between senile plaques and cerebrovascular amyloidosis in the brain of aged dogs. J Vet Med Sci. 1992; 54: 137-144.

11. Wegiel J, Wisniewski HM, Dziewiatowski J, Tarnawski M, Nowakowski J, Dziewiatowska A, Soltysiak Z. The origin of amyloid in cerebral vessels of aged dogs. Brain Research. 1995; 705: 225-234.

12. Yoshino T, Uchida K, Tateyama S, Yamaguchi R, Nakayama H, Goto N. A retrospective study of canine senile plaques and cerebral amyloid angiopathy. Vet Pathol. 1996; 33: 230-234.

13. Colle MA, Hauw JJ, Crespeau F, Uchihara T, Akiyama H, Checler F, Pageat P, Duykaerts C. Vascular and parenchymal $A \beta$ deposition in the aging dog: correlation with behavior. Neurobiology of Aging. 2000; 21: 695-704.

14. Jakel L, Van Nostrand WE, Nicoll JAR, Werring DJ, Verbeek MM. Animal models of cerebral amyloid angiopathy. Clinical Sciences. 2017; 131: 2469-2488.

15. Rodrigues LL, Mesquita LP, Costa RC, Gomes RG, Biihrer DA, Maiorka PC. Multiple infarcts and hemorrhages in the central nervous system of a dog with cerebral amyloid angiopathy: a case report. BMC Veterinary Research. 2018; 14: 370.

16. Fulkerson CV, Young BD, Jackson ND, Porter B, Levine JM. MRI characteristics of cerebral microbleeds in four dogs. Vet Radiol Ultrasound. 2012; 53: 389-393. 
406

407

408

409

410

411

412

413

414

415

416

417

418

419

420

421

422

423

424

425

426

427
17. Hodshon AW, Hecht S, Thomas WB. Use of the T2*-weighted gradient recalled echo sequence for magnetic resonance imaging of the canine and feline brain. Vet Radiol Ultrasound. 2014; 55: 599-606.

18. Kerwin SC, Levine JM, Budke CM, Griffin JF, Boudreau CE. Putative cerebral microbleeds in dogs undergoing magnetic resonance imaging of the head: a retrospective study of demographics, clinical associations, and relationship to case outcome. J Vet Intern Med. 2017; 31: 1140-1148.

19. Reis DJ, Golanov EV, Ruggiero DA, Sun MK. Sympatho-excitatory neurons of the rostral ventrolateral medulla are oxygen sensors and essential elements in the tonic and reflex control of the systemic and cerebral circulations. J Hypertens Suppl 1994; 12 : S159-S180.

20. Hasegawa D, Yayoshi N, Fujita Y, Fujita M, Orima H. Measurement of interthalamic adhesion thickness as a criteria for brain atrophy in dogs with and without cognitive dysfunction (dementia). Vet Radiol Ultrasound. 2005; 46: 452-457.

21. Noh D, Choi S, Choi H, Lee Y, Lee K. Evaluation of interthalamic adhesion size as an indicator of brain atrophy in dogs with and without cognitive dysfunction. Vet Radiol Ultrasound. 2017; 58: 581-587.

22. Landsberg GM, Nichol J, Araujo JA. Cognitive dysfunction syndrome: a disease of canine and feline brain aging. Vet Clin Small Anim. 2012; 42: 749-768.

23. Dewey CW, Davies ES, Xie H, Wakshlag JJ. Canine cognitive dysfunction: pathophysiology, diagnosis, and treatment. Vet Clin Small Anim. 2019; https://doi.org/10.1016/j.cvsm.2019.01.013. 
428

429

430

431

432

433

434

435

436

437

438

439

440

441

442

443

444

445

446

447

448

449

24. Dewey CW. Encephalopathies: disorders of the brain. In: Dewey CW, da Costa RC (eds): Practical Guide to Canine and Feline Neurology, $3^{\text {rd }}$ ed. Ames, IA; Wiley-Blackwell, 2016: 141-236.

25. Schutt T, Toft N, Berendt M. Cognitive dysfunction, progression of age-related behavioral changes, biomarkers, and survival in dogs more than 8 years old. J Vet Intern Med. 2015; 29: 1569-1577.

26. Estey CM, Dewey CW, Rishniw M, Lin DM, Bouma J, Sackman J, Burkland E. A subset of dog with presumptive idiopathic epilepsy show hippocampal asymmetry: a volumetric comparison with non-epileptic dogs using MRI. Front Vet Sci. 2017; DOI: 10.3389/fvets.2017.00183.

27. Leigh EJ, Mackillop E, Robertson ID, Hudson LC. Clinical anatomy of the canine brain using magnetic resonance imaging. Vet Radiol Ultrasound. 2008; 49: 113-121.

28. Chardimou A, Boulouis G, Gurol ME, Ayata C, Bacskai BJ, Frosch MP, Viswanathan A, Greenberg SM. Emerging concepts in sporadic cerebral amyloid angiopathy. Brain. 2017; 140: $1829-1850$.

29. Charidimou A. Elderly and forgetful with transient neurological spells: A story of two amyloids? Journal of the Neurological Sciences. 2015; 351: 1-2.

30. Caetano A, Ladeira F, Barbosa R, Calado S, Viana-Baptista M. Cerebral amyloid angiopathy-the modified Boston criteria in clinical practice. Journal of the Neurological Sciences. 2018; 384: 55-57.

31. Chapagain D, Range F, Huber L, Viranyi Z. Cognitive aging in dogs. Gerontology. 2018; 64: $165-171$. 
450

451

452

453

454

455

456

457

458

459

460

461

462

463

464

465

466

467

468

469

32. Rofina JE, van Ederen AM, Touissaint MJ, Secreve M, van der Spek A, van der Meer I, Van Eerdenburg FJ, Gruys E. Cognitive disturbances in old dogs suffering from the canine counterpart of Alzheimer's disease. Brain Res. 2006; 1069: 216-226.

33. Salvin HE, McGreevy PD, Sachdev PS, Valenzuela MJ. Under diagnosis of canine cognitive dysfunction: a cross-sectional survey of older companion dogs. Vet J. 2010; 184: 277-281.

34. Azkona G, Garcia-Belenguer SG, Chacon G, Rosado B, Leon M, Palacio J. Prevalence and risk factors of behavioural changes associated with age-related cognitive impairment in geriatric dogs. J Small Anim Pract. 2009; 50: 87-91.

9




\section{Figure Legends}

471 Figure 1. Transverse T2* gradient echo MR images demonstrating typical appearance of presumptive brain microhemorrhages. A-multiple microhemorrhages at the diencephalon level. B-a single microhemorrhage in the left cerebellum.

Figure 2. Transverse T2-weighted MR image depicting method of measuring interthalamic adhesion height (thickness).

Figure 3. Mid-sagittal T2-weighted image depicting method of measuring interthalamic adhesion area.

Figure 4. Box and whisker plots of interthalamic adhesion area indexed to total brain

Figure 5. Box and whisker plots of microhemorrhage numbers for control dogs, spontaneous brain microhemorrhage dogs without cognitive dysfunction (SBM) and dogs with cognitive dysfunction (CCD).

Figure 6. Scatter plots of normalized interthalamic sagittal adhesion height (A) and area (B) against age in control dogs (black circles), dogs with spontaneous brain microhemorrhages (open circles) and dogs with cognitive dysfunction (triangles) 


\section{Figure 1}

Typical examples of microhemorrhages on T2* weighted images

Transverse T2* gradient echo MR images demonstrating typical appearance of presumptive brain microhemorrhages. (A) Multiple microhemorrhages at the diencephalon level. (B) A single microhemorrhage in the left cerebellum.

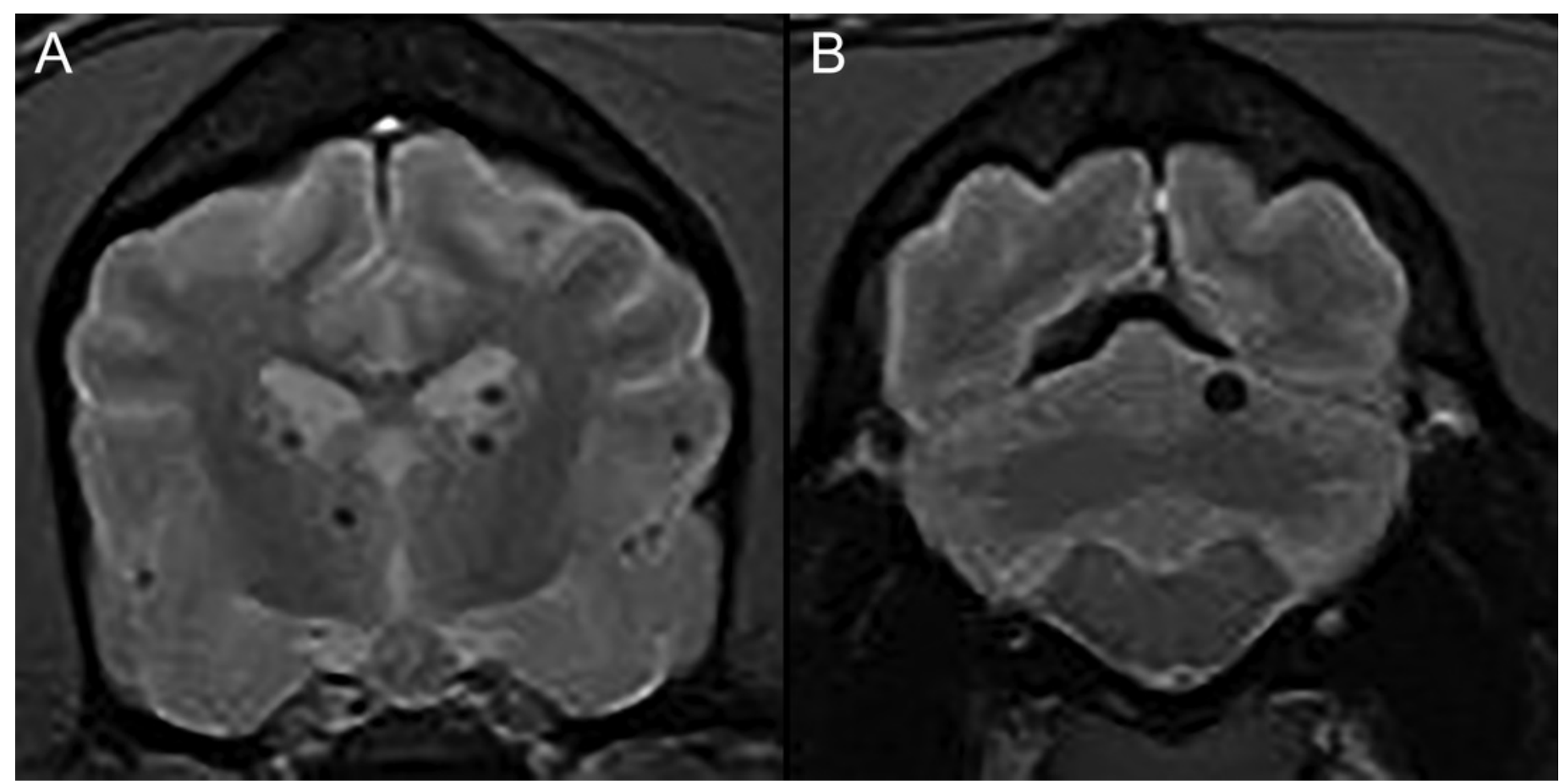




\section{Figure 2}

Method for measuring interthalamic adhesion height from a transverse T2 weighted image.

Transverse T2-weighted MR image depicting method of measuring interthalamic adhesion height (thickness).

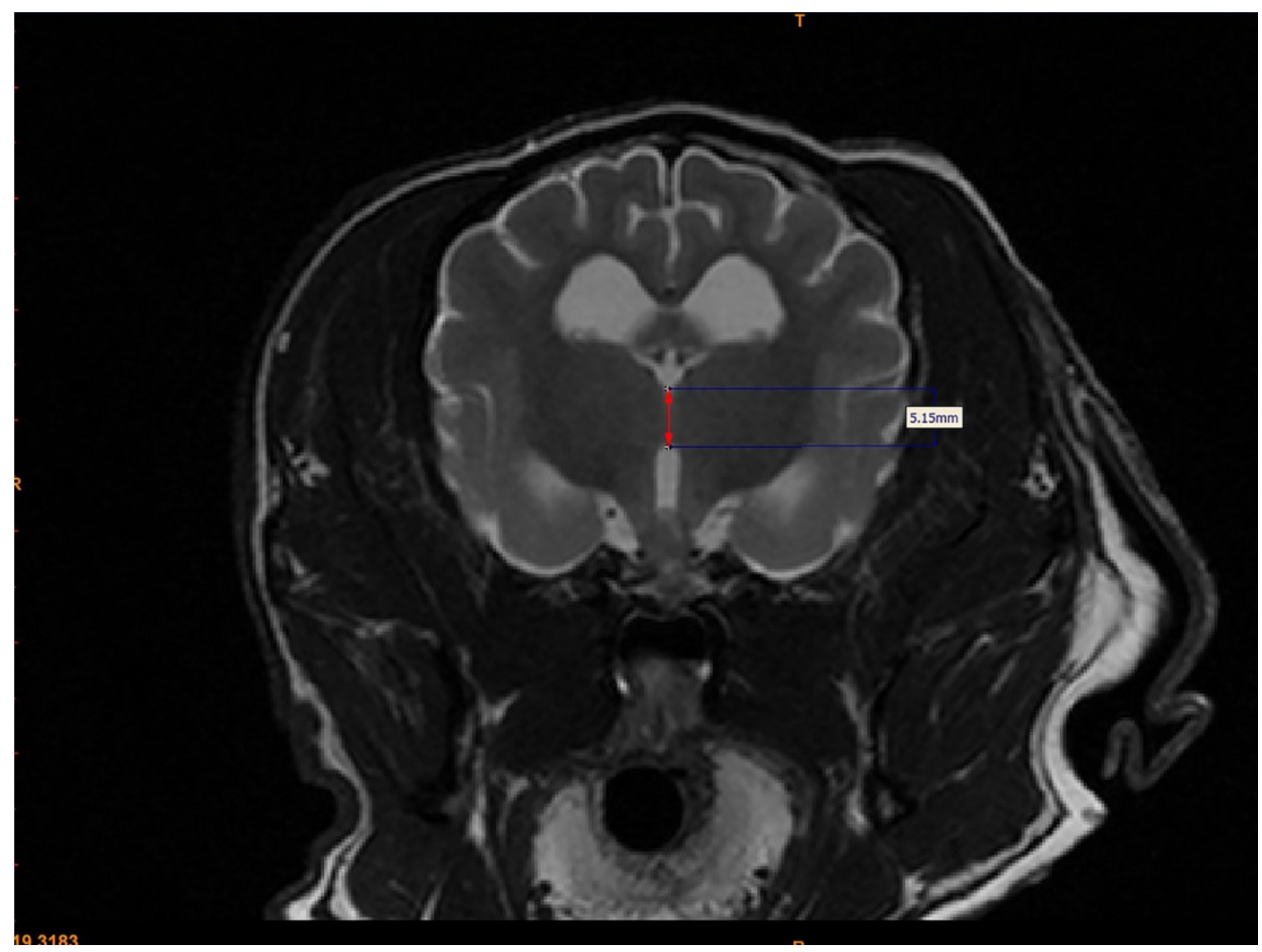


Figure 3

Method of measuring interthalamic adhesion area from a mid-sagittal T2 weighted image

Mid-sagittal T2-weighted image depicting method of measuring interthalamic adhesion area. 


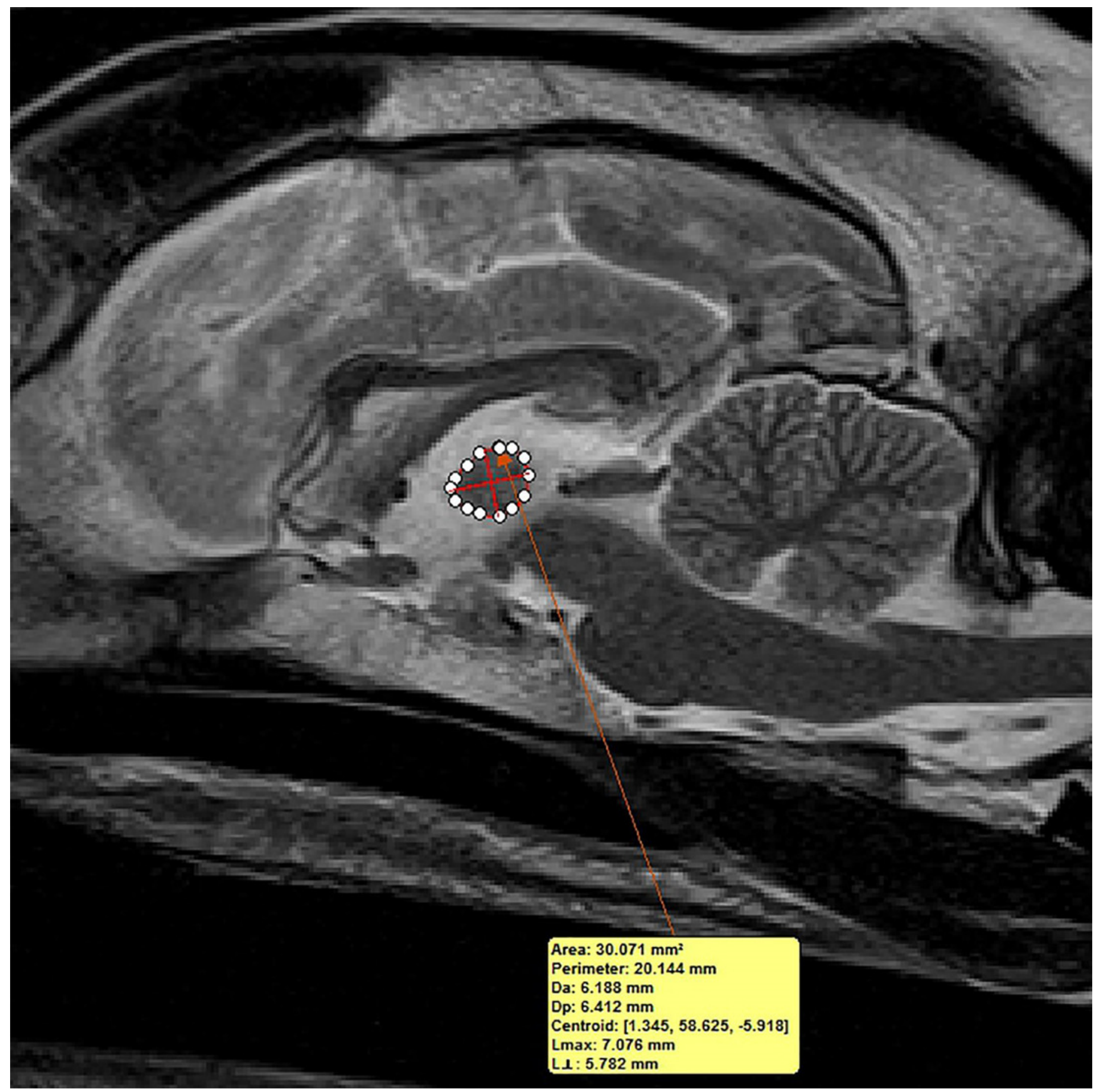


Figure 4

Box and whisker plots of interthalamic adhesion areas normalized to total brain volume for three groups of dogs.

Box and whisker plots of interthalamic adhesion area indexed to total brain volume (TBV) for control dogs, spontaneous brain microhemorrhage dogs without cognitive dysfunction (SBM) and dogs with cognitive dysfunction (CCD).
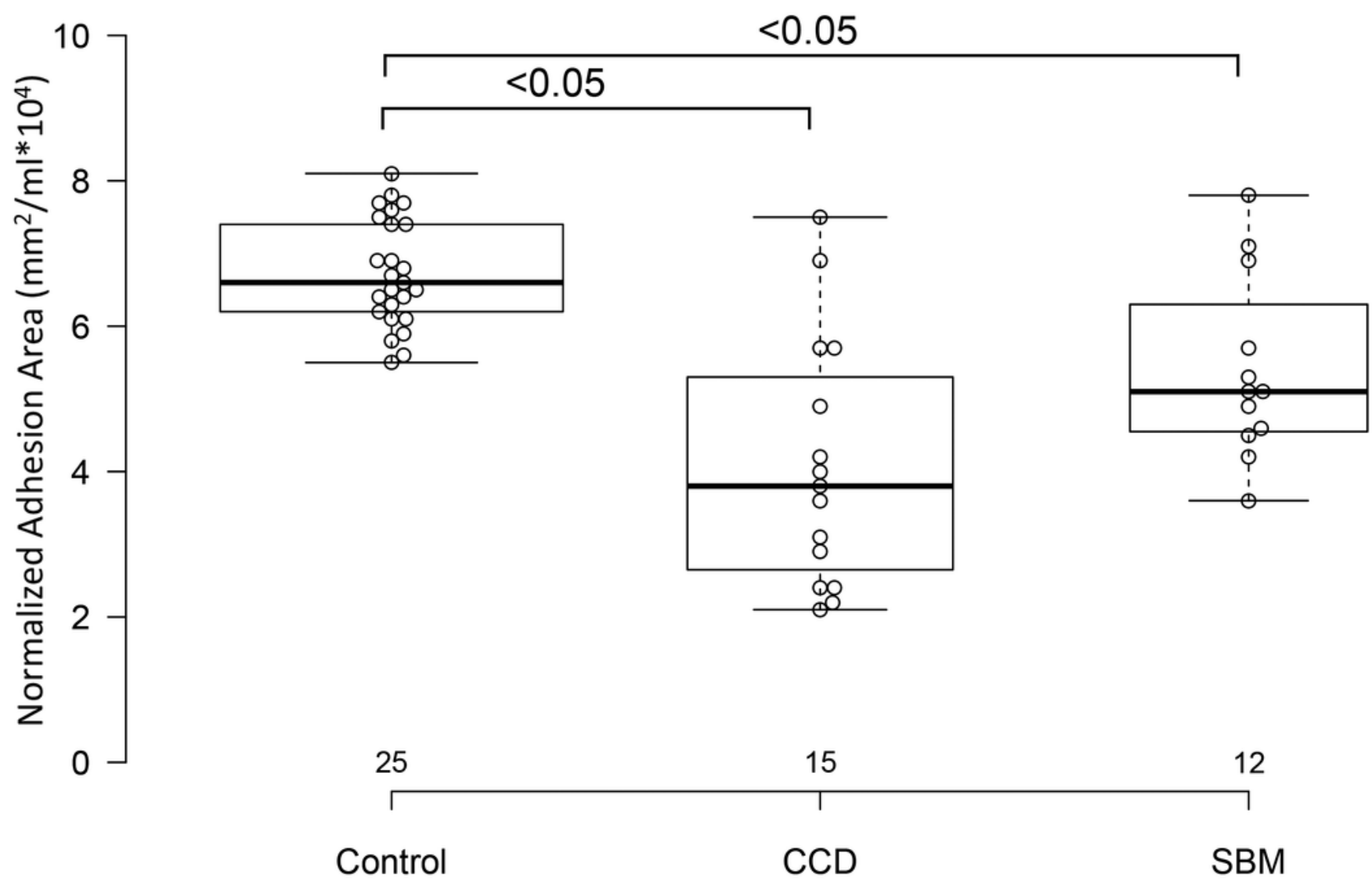
Figure 5

Box and whisker plots of microhemorrhage numbers for three groups of dogs.

Box and whisker plots of microhemorrhage numbers for control dogs, spontaneous brain microhemorrhage dogs without cognitive dysfunction (SBM) and dogs with cognitive dysfunction (CCD).

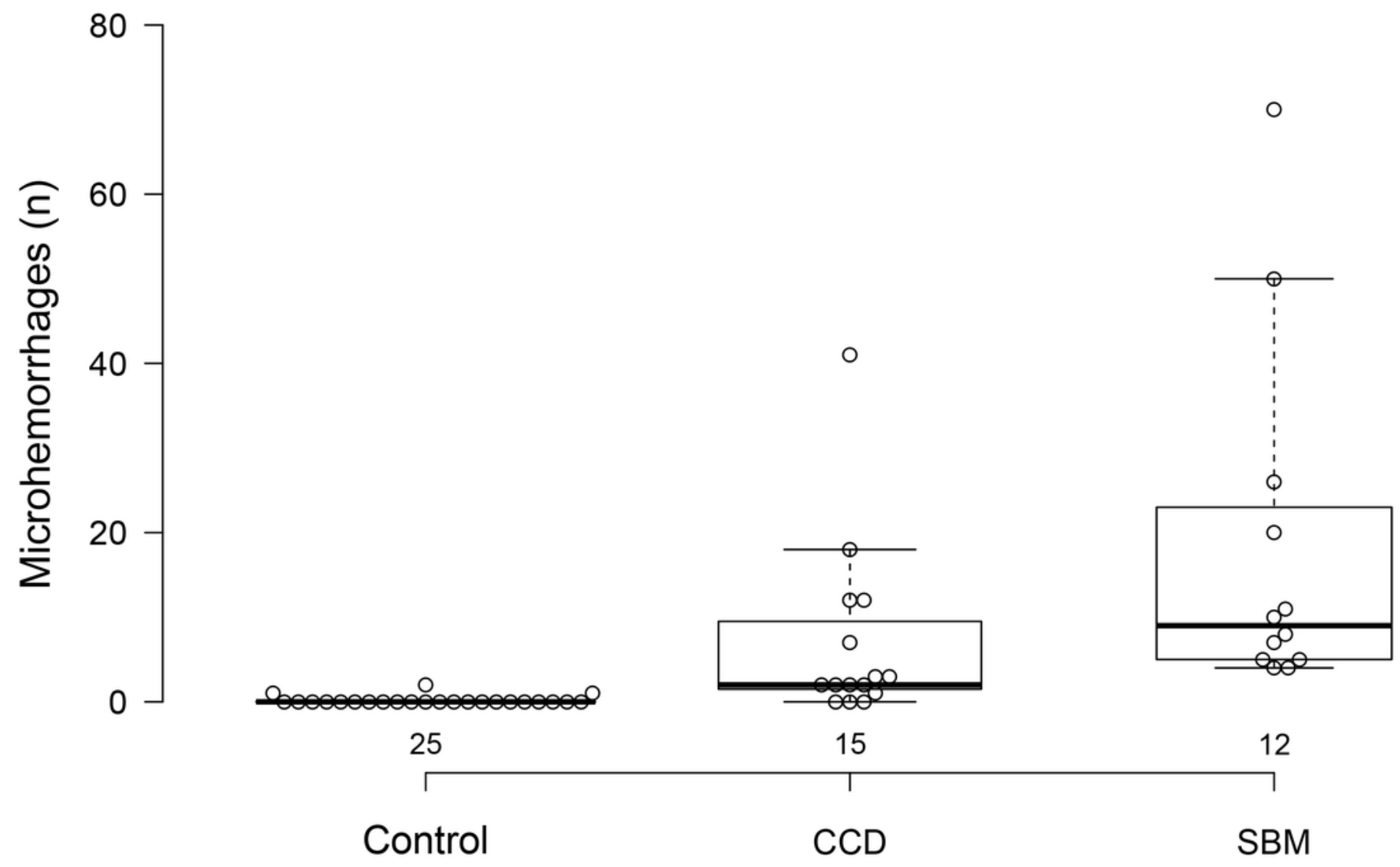


Figure 6 (on next page)

Scatterplots of interthalamic adhesion measurements across age.

Scatter plots of normalized interthalamic sagittal adhesion height (A) and area (B) against age in control dogs (black circles), dogs with spontaneous brain microhemorrhages (open circles) and dogs with cognitive dysfunction (triangles) 


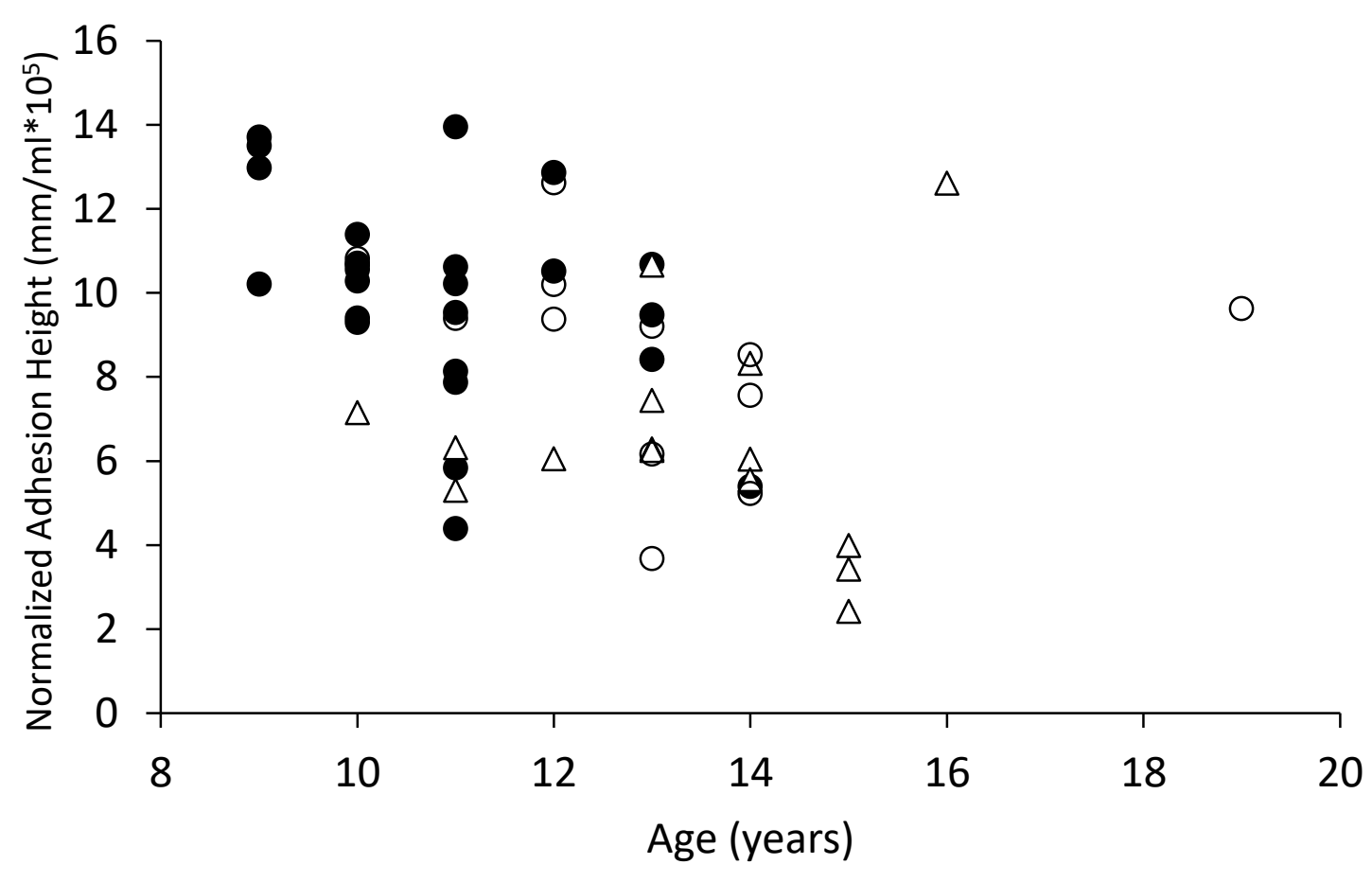




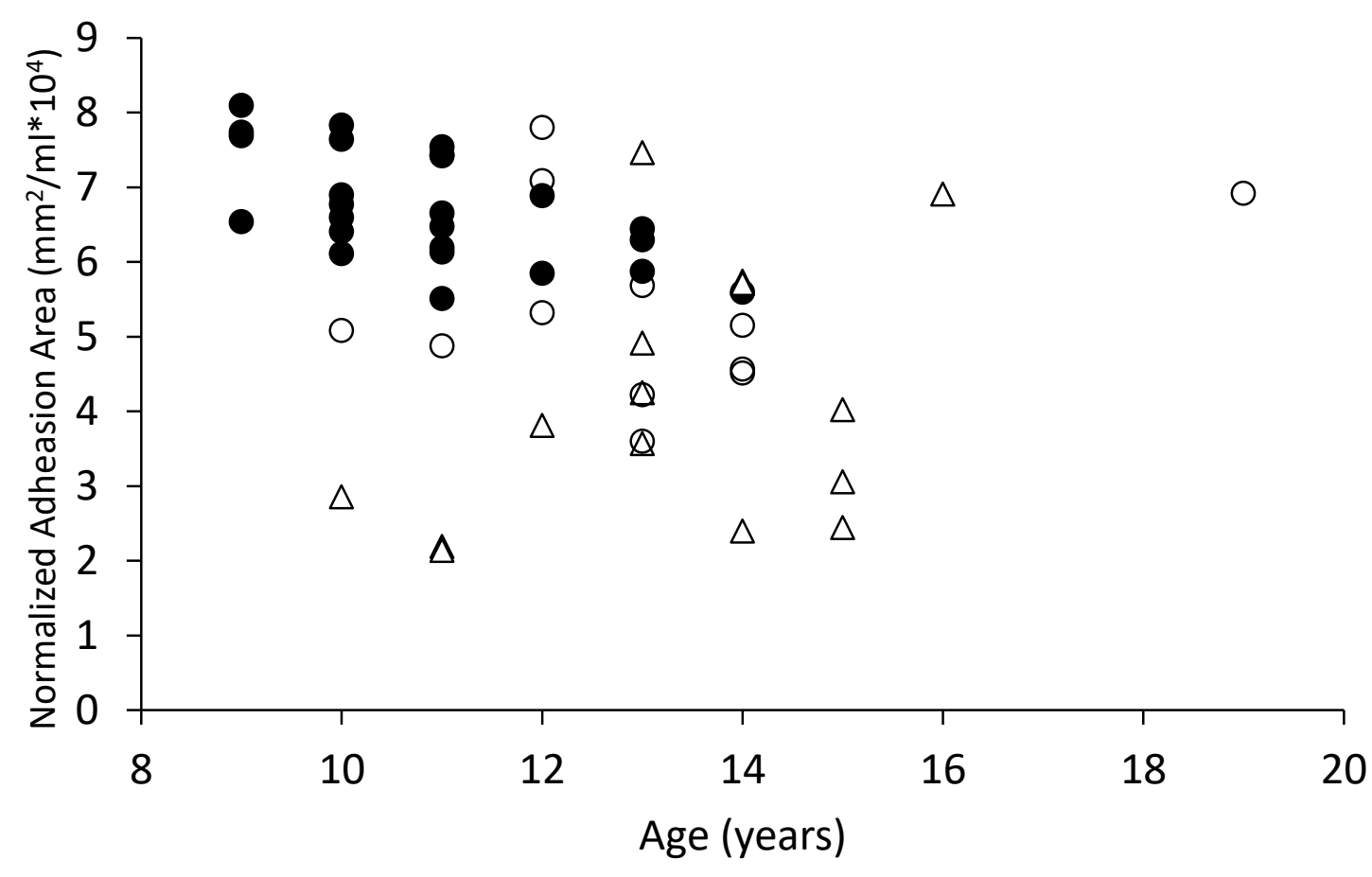

